\title{
Finite-lattice expansion for Ising models on quasiperiodic tilings
}

\author{
Przemyslaw Repetowicz \\ Department of Mathematics, Heriot-Watt University,Riccarton, Edinburgh EH14 4 AS, United Kingdom
}

(November 1, 2018)

\begin{abstract}
Low-temperature series are calculated for the free energy, magnetisation, susceptibility and field-derivatives of the susceptibility in the Ising model on the quasiperiodic Penrose lattice. The series are computed to order 20 and estimates of the critical exponents $\alpha, \beta$ and $\gamma$ are obtained from Padé approximants.
\end{abstract}

\section{INTRODUCTION}

The problem of the relevance of disorder for phase transitions in lattice models of statistical mechanics has attracted attention for many years and the discovery of quasicrystals [1] has served to increase interest in the physical properties of disordered systems. A fundamental problem in this field is whether quasiperiodic order is strong enough to change the critical behaviour of magnetic phase transitions. To investigate this problem we consider in this article a classical Ising model defined on an underlying quasiperiodic lattice.

There have been many works in this field since the late eighties. A heuristic criterion (Harris-Luck criterion) has been formulated [2] which relates the critical behaviour to fluctuations of the number of spin couplings in a given region. The spatial scaling of fluctuations was described in terms of a "wandering exponent" $\omega$ which was required to exceed a threshold $\omega_{c}$ in order to produce a new universality class. For the majority of quasiperiodic structures existing in reality, such as the structural models of quasicrystalline phases discovered so far $\omega$ can be calculated exactly, due to the self-similarity or inflational symmetry of the structure, yielding a value smaller than the threshold and suggesting the irrelevance of disorder. However since numerous structures like the rhombic sevenfold or ninefold lattices [3, th exist, which are deprived of inflational symmetry and are therefore potential candidates for novel critical behaviour, there is still a strong motivation for dealing with quasiperiodic Ising models.

Quasiperiodic Ising models were investigated by Monte-Carlo simulations [5 \&] which at present, seem to yield the most precise estimates for the transition temperature and critical exponents. Indeed, in [6] computations for large periodic approximants (PA) of the Penrose tiling (PT) 9 were carried out and obtained values for the correlation length $\nu$ and the two-spin correlation function $\eta$ exponents with two-digit precision $(\nu=1.02 \pm 0.02, \eta=0.252 \pm 0.003)$ which agreed with the square lattice values $(\nu=1, \eta=0.25)$. Moreover, the non-universal critical temperature $T_{c}$ has also been determined with an impressively small error $k T_{c}=2.398 \pm 0.003$.

It is worth mentioning that a novel invaded-cluster algorithm, which modifies the temperature during the simulation towards the critical one, as opposed to standard Monte-Carlo algorithms with fixed temperature, was also applied to quasiperiodic systems [8] to give an improved estimate of $T_{c}$. The critical exponents are not available in this case, however.

Another approach is an approximate renormalisation group analysis 10 11 which yields poor results, however. For the PT the specific heat exponent equals $\alpha=-0.1083$ versus $\alpha=0$ for the square lattice.

Quasiperiodic Ising models were also examined by graphical expansion methods [12,13] and by calculating exact partition functions for PA, obtained from the Kac-Ward determinant |14]. In the first case estimates for $T_{c}$ and critical exponents have not been considerably improved but this approach demonstrated a new feature, a very slow convergence of the partition function $(Z)$ series to its predicted asymptotic form. We also investigated the set of zeros of $Z$ in the complex plane (Fisher zeros), which turned out to be much more complicated than in the square lattice case.

The Kac-Ward determinant method appeared to yield highly accurate estimates of the critical temperature of quasiperiodic Ising models (for example $k T_{c}=2.397820(7)$ for the PT). Moreover, within this framework it was possible to construct a two dimensional Ising model with relevant fluctuations, i.e. for which $\omega>\omega_{c}$, which shows up another novel feature, namely the divergence of high temperature series [16]. This example is interesting because it points out that in some cases the reliability of methods for extracting critical values from analysis of a series expansion, like the Padé- or differential-approximants methods, [15] can be questioned. An inspection of the Fisher zeros furnished the explanation, since it appeared that the moduli of some complex zeros were smaller than the modulus of the physical singularity 
(real zero) and thus the complex zeros, rather than the real zero, were limiting the region of convergence the series.

In this paper it is not our purpose to improve on estimating $T_{c}$ or $\alpha$ for quasiperiodic Ising models since, due to the slow convergence of series expansions [14 a large, inaccessible number of terms is needed to make progress in this field. Instead, we aim at generalising the series expansion approach to the case of non-zero field quasiperiodic Ising or Potts models [17] and provide alternative estimates of the magnetic exponents, $\beta$, and $\gamma$. Moreover, this approach allows us to investigate the problem of a disorder-driven "softening" of the first-order phase transition in $Q$-state Potts models for $Q>4[18$.

\section{THE FINITE LATTICE EXPANSION METHOD FOR ISING MODELS}

The problem consists in calculating the partition function $Z(\mathcal{G})$ of an Ising model on a lattice $\mathcal{G}$ by series expansion. The partition function with field $B$ and coupling constant $J$ is defined in the usual way:

$$
Z(\mathcal{G})=\sum_{\left\{\sigma_{j}\right\}} \exp \beta\left\{-J \sum_{\langle j, k\rangle} \Delta\left(\sigma_{j}, \sigma_{k}\right)-B \sum_{j=1}^{N} \Delta\left(\sigma_{j}, 0\right)\right\} \quad \text { where } \quad \Delta\left(\sigma_{1}, \sigma_{2}\right)=\left\{\begin{array}{cc}
0 & \sigma_{1}=\sigma_{2} \\
1 & \text { otherwise }
\end{array}\right.
$$

where the sum over spin configurations $\left\{\sigma_{j}\right\}=\left\{\sigma_{1}, \sigma_{2}, \ldots, \sigma_{N}\right\}$ consists of $N$ sums each of which runs over $\sigma_{j}=\{1,2\}$. Starting from cluster integral theory (page 42-46 and page 73 in [19]) one can formulate a free energy (F) expansion in terms of connected graphs for a wide range of models from statistical mechanics. In particular, for the non-zero field Ising model or the $Q$-state Potts models (the generalisation of the former one with $Q$ values of spin at each site) the expansion on a lattice $\mathcal{G}$ reads

$$
\log Z(\mathcal{G})=\sum_{r}\left(C_{r} ; \mathcal{G}\right) k_{r}(w) \quad \text { where } \quad w=\tanh \beta J
$$

where the sum on the right-hand side runs over connected graphs $C_{r}$ from $\mathcal{G}$. The quantity $\left(C_{r} ; \mathcal{G}\right)$ denotes the embedding number of $C_{r}$ in $\mathcal{G}$, counting the number of ways $C_{r}$ can be embedded in $\mathcal{G}$. Finally, the weight functions $k_{r}(w)$ depend only on $C_{r}$ not on $G$. Making use of the independence of weights $k_{r}(w)$ from the lattice we can write equation (2) substituting each connected graph $C_{r}$ for $\mathcal{G}$, solve the system of equations for the weights and plug in the results to equation (2). We obtain

$$
\log Z(\mathcal{G})=\sum_{g_{r}} a_{r} \log Z\left(g_{r}\right)
$$

where $a_{r}=\sum_{p}\left(g_{p} ; G\right) b_{r, p}$ and $b_{r, p}$ is inverse to the matrix of embedding numbers, i.e. $b_{r, p}=\left(g_{r} ; g_{p}\right)^{-1}$. The sum on the right-hand side in (3) runs over $g_{r}$ from a subset of all connected graphs. It turns out [20] that the graph $g_{r}$ can furnish a non-vanishing contribution, i.e. $a_{r} \neq 0$, if and only if it is an overlap of the embeddings of two other graphs having non-vanishing contributions. The construction of graphs therefore runs as follows; we start from several "fairly large" graphs and construct all possible overlaps of their embeddings in the lattice in a recursive way. This limits the number of contributing graphs considerably, when compared to expansion (2), but, except for regular lattices like the square or honeycomb lattice, still leaves the problem of determining $g_{r}$ and the contributions $a_{r}$ open. Indeed, for the square lattice where $g_{r}$ are rectangles, $a_{r}$ can be explicitly expressed via the ratio of the graph side lengths [21] and the order to which the expansion is correct is in direct connection with the perimeter length of the largest graphs under consideration. For the quasiperiodic lattices which we wish to investigate the problem is not so simple. In what follows we focus on the PT [9] and present the details of the expansion method for it in the next section.

\section{CALCULATION OF SERIES EXPANSION FOR THE PENROSE TILING}

The PT is an aperiodic tiling of a plane by two kinds of rhombi of unit length side with angles $2 \pi / 5$ and $4 \pi / 5$ respectively. A discussion of the methods of generation and geometrical properties of this tiling can be found in [13, here we only mention a particularly useful feature, namely that embedding numbers of finite patches from this tiling can be calculated exactly and take the form $n+m \tau$ where $\tau=(\sqrt{2}+1) / 2$ is the golden number and $n, m$ are rational numbers. The calculation of the series expansion consists therefore of the following steps: 
1. Choose an initial set of "fairly large" graphs which are expected to be large enough that every connected subgraph of the underlying tiling with perimeter length not larger than a given threshold $2 L$ can be embedded in one of them. While on the square lattice this condition is satisfied by all possible rectangles with perimeter length $2 L$ on the PT the things are worse due to the lack of periodicity of the tiling. Moreover, as opposed to the square lattice graphs in the PT can have different "boundary line fillings", i.e. there are different graphs having the same boundary line [13]. Knowing that the PT contains eight different vertex types, i.e. different site environments related to the nearest neighbours, we cut out appropriately large patches around each vertex type, obtaining eight patches, and found all possible "boundary line fillings". There is still a lot of ambiguity in this procedure since a patch in not uniquely determined by the vertex type of its central site. It would be more correct to take all possible higher order vertex types [22], i.e. $m$-order vertex types related to neighbours located not further than $m$ edges lengths from the site, but since their number grows quite rapidly with the order, the initial set of graphs would be too numerous and the generation of overlaps (see next item) too time consuming.

2. Generate all possible overlaps of embeddings of the initial graphs in the tiling.

It is difficult to estimate how the time of the generation depends on the number of initial graphs. Let us say a couple of words about this, however. We group graphs into generations so that the initial set of graphs constitutes the zeroth generation and the nth generation consists of overlaps of graphs from the (n-1)st and zeroth generations. Since the time for creating the nth generation depends on the product of numbers of graphs from generation zero, $\# g(0)$ and generation $(n-1)$, $\# g(n-1)$, starting from a too numerous zeroth generation should be avoided. The total number of overlaps grows rather slowly with $\# g(0)$ for large $\# g(0)$ and most of the computing time will be devoted to checking and rejecting graphs which occurred before. On the other hand if we took too few initial patches, the covering of the lattice with them would be incomplete, there would be plenty of "holes" not covered by any of the patches, and thus the series expansion would be error laden. The rule of thumb is to take $g(0)$ not larger than twenty and choose the patches in such a way that their interiors differ as much as possible.

Again, on the square lattice it's immediately clear that the overlaps are rectangles, because every rectangle can be constructed as an overlap of two other rectangles, whereas on the PT the shapes of graphs and their quantity depends on the initial set. To make the things worse we are not even sure that we obtain star graphs (page 1-16 in 19), i.e. graphs without articulation points, because the initial graphs are not necessarily convex. Connected graphs consisting of multiple components will cause some difficulties by the calculation of partition functions by the transfer matrix method (see following items).

3. Calculate the contribution $a_{r}$ of graph $g_{r}$ in (3) in the following recursive way

$$
a_{r}=\left(g_{r} ; G\right)-\sum_{r \in p} a_{p}\left(g_{r} ; g_{p}\right)
$$

where the sum on the right-hand side runs over all graphs $g_{p}$ in which $g_{r}$ can be embedded.

4. Calculate $\log$ arithms of partition functions $\log Z\left(g_{r}\right)$ by the transfer-matrix method.

The transfer-matrix method

Here we have to distinguish two cases, namely the case when the graph has no articulation points (star graph) and the contrary (multicomponent graph). The latter is undoubtedly more complicated but fortunately it turns out that it takes place only in a minority of the graphs under consideration. Let us firstly discuss the case of a star graph. We can define a perimeter of the graph, i.e. a line consisting of edges each of which belongs only to one rhombi. The sum over spin configurations can be performed by moving a boundary line across the graph. At each stage the boundary line goes through a number, say $k$, sites. For the $Q$-state Potts model we have $Q^{k}$ different spin configurations on the boundary line. Now we define a $Q^{k}$ dimensional vector $Z(\sigma)$ consisting of partition functions calculated for the patch composed of sites from the boundary line, with a given spin configuration $\sigma=\left\{\sigma_{1}, \sigma_{2}, \ldots, \sigma_{N}\right\}$ assigned to them, and sites already traversed by the boundary line. The initial values of $Z(\sigma)$ are given by:

$$
Z(\sigma)=\tilde{x}^{a}(1-\tilde{y})^{b} \quad \text { where } \quad \tilde{x}=\exp (-\beta J), \quad \tilde{y}=1-\exp (-\beta B / 2)
$$

and 


$$
a=\sum_{p=1}^{N-1} \Delta\left(\sigma_{p}, \sigma_{p+1}\right), \quad b=2 \sum_{p=1}^{N} \Delta\left(\sigma_{p}, 0\right)
$$

Shifting the boundary line corresponds to generating a new vector $Z^{\prime}\left(\sigma^{\prime}\right)$ of partition functions from the old vector $Z$. There is a lot of ambiguity in shifting the boundary line by a given number of tiles. In our case it amounts, however, to considering only three kinds of movements, by one tile, by two tiles and a shift between two given boundary line configurations, which we discuss in the following. Placing the initial boundary line on the perimeter of the graph and moving it at each stage by certain number of tiles, see FIG.11, we have performed the sum over all configurations after reaching the final position of the boundary line (also lying on the perimeter).
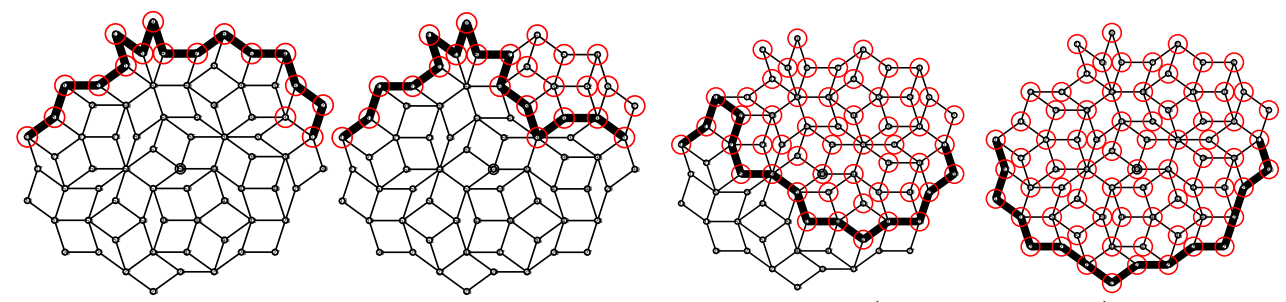

FIG. 1. Shifting of a boundary line through a graph, from the initial (leftmost picture) to the final (rightmost picture), corresponding to calculating the partition function by transfer-matrix method.

Now we discuss the details of updating the partition functions for the two kinds of boundary line movements, see FIG.2.

1. One-tile movement

For $1 \leq J \leq N$ we have

$$
Z^{\prime}\left(\sigma^{\prime}\right)=\tilde{x}^{a}(1-\tilde{y})^{b} \sum_{\sigma_{J}=1}^{q} Z(\sigma)
$$

where $a=\Delta\left(\sigma_{J-1}^{\prime}, \sigma_{J}^{\prime}\right)+\Delta\left(\sigma_{J}^{\prime}, \sigma_{J+1}^{\prime}\right)$ and $b=f \Delta\left(\sigma_{J}^{\prime}, 0\right), f=2$.

\section{Two-tiles movement}

For $1 \leq L<P \leq N$ we have

$$
Z^{\prime}\left(\sigma_{\rho}^{\prime}\right)=\tilde{x}^{a}(1-\tilde{y})^{b} \sum_{\sigma_{P+1}=1}^{q} \sum_{\sigma_{P+2}=1}^{q} Z(\sigma)
$$

where $a=\Delta\left(\sigma_{L}^{\prime}, \sigma_{P+1}^{\prime}\right)+\Delta\left(\sigma_{P+1}^{\prime}, \sigma_{P+2}^{\prime}\right)+\Delta\left(\sigma_{P+2}^{\prime}, \sigma_{L+1}^{\prime}\right)+\Delta\left(\sigma_{P}^{\prime}, \sigma_{P+3}^{\prime}\right)$ and $b=f_{1} \Delta\left(\sigma_{P+1}^{\prime}, 0\right)+$ $f_{2} \Delta\left(\sigma_{P+2}^{\prime}, 0\right)$ where $f_{1}=f_{2}=2$ and the new spin configuration is permuted with respect to the old one $\sigma_{\rho}^{\prime}=\left\{\sigma_{\rho_{1}}^{\prime}, \sigma_{\rho_{2}}^{\prime}, \ldots, \sigma_{\rho_{N}}^{\prime}\right\}$ and

$$
\rho_{p}=\left\{\begin{array}{rl}
p & p \leq L \\
P+1 & p=L+1 \\
P+2 & p=L+2 \\
p-2 & p \geq L+3
\end{array}\right.
$$

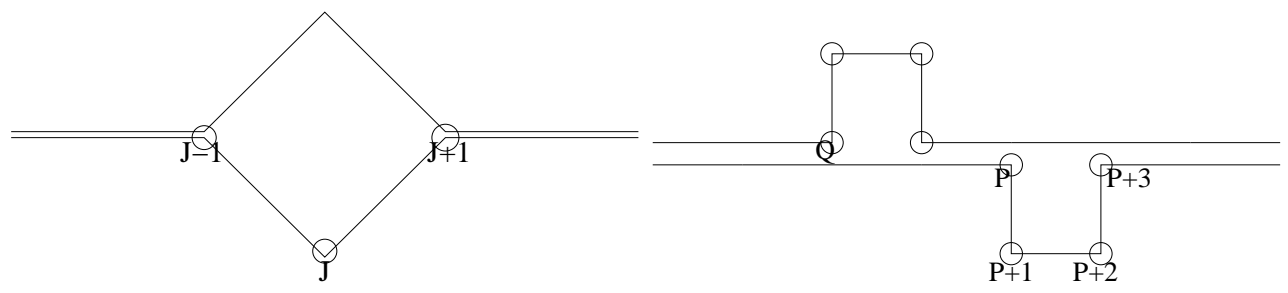

FIG. 2. Two kinds of movements of the boundary line, by one tile (left) and by two tiles (right). 


\section{Shifting the boundary line to the final position}

In most cases it is possible to displace the boundary line from the initial to the final position by a sequence of the movements defined above. Sometimes, however, we arrive in a dead end because none of the movements can be done, see FIG.3. In this case we have to shift the line directly to its final position by summing over all the spins which have not been taken into account yet. The formal prescription for updating $Z(\sigma)$ in this case reads:

$$
Z^{\prime}\left(\sigma_{F_{1}}, \ldots, \sigma_{F_{9}}\right)=\sum_{\sigma_{s_{1}}, \sigma_{s_{2}}, \sigma_{s_{3}}} \tilde{x}^{a}(1-\tilde{y})^{b} Z\left(\sigma_{B_{1}}, \ldots, \sigma_{B_{9}}\right)
$$

where $a=\sum_{j=1}^{6} \Delta\left(\sigma_{e_{j, 1}}, \sigma_{e_{j, 2}}\right)$ and $b=2 \sum_{j=1}^{3} \Delta\left(\sigma_{s_{j}}, 0\right)$ and $B=\{1,16,15,14,13,18,19,20,9\}, F=$ $\{1,16,15,14,13,12,11,10,9\}, e=\{(9,10),(10,11),(11,12),(12,13),(12,9),(9,18)\}$ and $s=\{12,11,10\}$ denote the current and the final boundary lines, the edges and the sites which were not taken into account yet respectively.
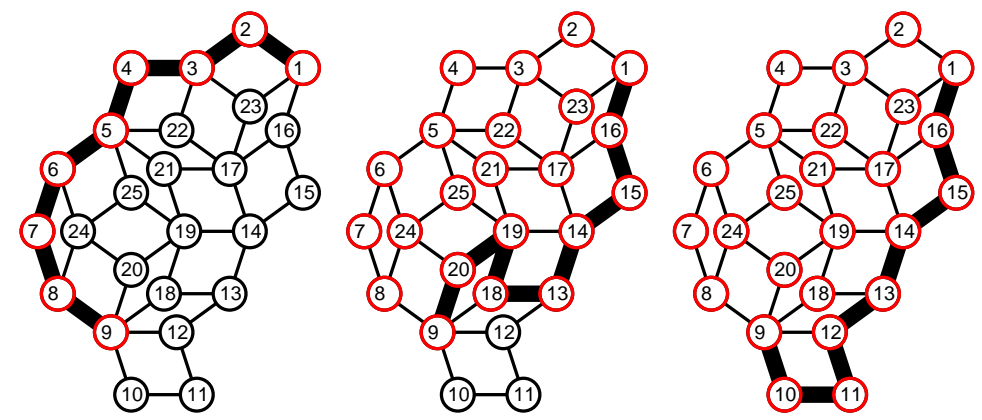

FIG. 3. A boundary line (middle picture) which cannot be pushed forward by performing one of the movements discussed above. The initial and the final line configuration are shown at the left and at the right respectively.

Can the transfer-matrix formalism (tmf) also be applied to the case of a multicomponent graph? The answer is affirmative because every connected graph can be dissected into its star graph components for which the tmf is applicable. Since, however, star graph components share certain sites at their boundaries, which we call in the following isolated sites, we have to calculate a whole set of partition functions with given spin values at isolated sites and combine them to get the partition function of the whole graph. In the following we assume the simplest case namely, that every isolated site is shared by exactly two star components. This was indeed the case by our overlap graphs. Let us explain the procedure for the case of a graph depicted in FIG.4. The partition function $Z$ can be build up from partition functions $Z_{A}\left(\sigma_{1}\right)$, $Z_{B}\left(\sigma_{1}, \sigma_{2}, \sigma_{3}\right), Z_{C}\left(\sigma_{3}\right)$ and $Z_{D}\left(\sigma_{2}\right)$ corresponding to star components $A, B, C$, and $D$ with isolated spins $\sigma_{1}, \sigma_{2}$ and $\sigma_{3}$.

$$
Z=\sum_{\sigma_{1}, \sigma_{2}, \sigma_{3}} Z_{A}\left(\sigma_{1}\right) Z_{B}\left(\sigma_{1}, \sigma_{2}, \sigma_{3}\right) Z_{C}\left(\sigma_{3}\right) Z_{D}\left(\sigma_{2}\right)
$$
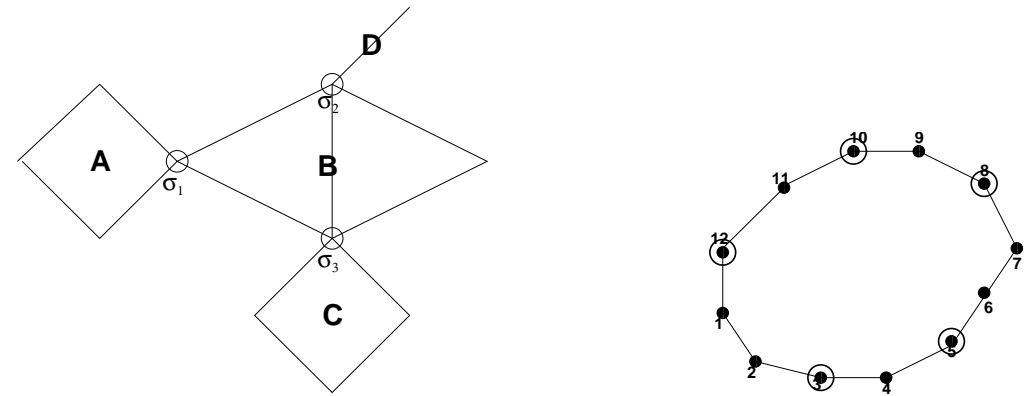

FIG. 4. A multicomponent graph consisting of four star components (left) and the perimeter of a star graph with two isolated sites on the initial boundary line (consisting of sites from 1 to 7 ) and three isolated sites on the final boundary line (sites from 7 to 12) (right). The isolated sites are marked with circles.

Now, the problem consists in calculating partition functions for a star graph with specified spins at isolated sites located at the boundary. Assume that we have $p$ isolated sites $j_{k}, k=1, . ., p$ located at the 
initial boundary line and $q$ isolated sites $l_{k}, k=1, . ., q$ at the final boundary line respectively, see FIG. 4 . The calculation of $Z\left(s_{j_{1}}, \ldots, s_{j_{p}}, s_{l_{1}}, \ldots, s_{l_{q}}\right)$ amounts to repeating the $\operatorname{tmf} Q^{p+q}$ times and modifying the initial and the final partition function set by setting certain entries to zero. We replace the initial partition function set by

$$
\left[\prod_{k=1}^{p} \delta\left(\sigma_{j_{k}}, s_{j_{k}}\right)\right] Z\left(\sigma_{1}, \ldots, \sigma_{N}\right) \text { for given } s_{j_{1}}, \ldots, s_{j_{p}}
$$

and the final partition function set is multiplied by $\left[\prod_{k=1}^{q} \delta\left(\sigma_{l_{k}}, s_{l_{k}}\right)\right]$ again for a given spin configuration $s_{l_{1}}, \ldots, s_{l_{q}}$. Another slight modification which is required consists in setting the factors $f, f_{1}$ and $f_{2}$ entering in the exponent $b$ in equations (7) according to whether the site is isolated (one) or not (two).

\section{SERIES EXPANSION OF THE FREE ENERGY, MAGNETISATION AND FIELD DERIVATIVES OF THE MAGNETISATION}

We have performed calculations for a set of graphs constructed in the following way. We cut off seven fairly round shaped patches from the PT so that the central sites of the patches had different vertex types and their perimeter lengths were not larger than 30 edge lengths. Then we enlarged the set of patches by all possible "boundary line fillings" obtaining in effect twelve patches, see FIG.5. In the next step we constructed all possible graphs contributing to the expansion in the recursive way described in section III. Their number turned out to be 1004. This part of computations was rather tedious, up to two weeks for the second set on a SunOS machine, because in generating graph overlaps many graphs turned up repeatedly and had to be rejected. In the next step we generated "decorations" of graphs, i.e. we determined vertex types of all sites of the graph including those on its boundary. Since the graphs could have several decorations the number of graphs we have to deal with increased to 5737 . Now we were ready to compute the coefficients $a_{r}$ entering in (3) which appeared to be different from zero only for a small fraction of all graphs, namely for 154 graphs. This is not a surprising result since on the square lattice the vast majority of rectangles used in the expansion yields zero coefficients as well [21]. Fortunately, most of the relevant graphs here were star graphs so we could easily compute the free energies $\log Z\left(g_{r}\right)$ entering in (3) in the way described in section [II]. There were however some awkward multicomponent graphs for which partition function computations were more tedious. The series expansion is shown beneath.

After reordering the expansion (3), i.e. collecting together terms with the same power of $\tilde{y}$, the free energy $F(\tilde{x}, \tilde{y})$ takes the form.

$$
F(\tilde{x}, \tilde{y})=\log Z(\mathcal{G})=F_{0}(\tilde{x})+F_{1}(\tilde{x}) \tilde{y}+F_{2}(\tilde{x}) \tilde{y}^{2}+\ldots=\sum_{n=0}^{\infty} F_{n}(\tilde{x}) \tilde{y}^{n}
$$

Quantities like the spontaneous magnetisation $M(\tilde{x})$, susceptibility $\chi(\tilde{x})$ and field derivatives of the susceptibility $\chi^{(n)}(\tilde{x})=d^{n} \chi(\tilde{x}) / d \tilde{y}^{n}$ can be expressed as linear combinations of the polynomials $F_{n}(\tilde{x})$.

$$
\begin{aligned}
M(\tilde{x}) & =d F(\tilde{x}, \tilde{y}) /\left.d B\right|_{B=0}=F_{1}(\tilde{x}) \\
\chi(\tilde{x}) & =d^{2} F(\tilde{x}, \tilde{y}) /\left.d B^{2}\right|_{B=0}=2 F_{2}(\tilde{x})-F_{1}(\tilde{x}) \\
\chi^{(1)}(\tilde{x}) & =d^{3} F(\tilde{x}, \tilde{y}) /\left.d B^{3}\right|_{B=0}=6 F_{3}(\tilde{x})-6 F_{2}(\tilde{x})+F_{1}(\tilde{x}) \\
\chi^{(2)}(\tilde{x}) & =d^{4} F(\tilde{x}, \tilde{y}) /\left.d B^{4}\right|_{B=0}=24 F_{4}(\tilde{x})-36 F_{3}(\tilde{x})+14 F_{2}(\tilde{x})-F_{1}(\tilde{x})
\end{aligned}
$$



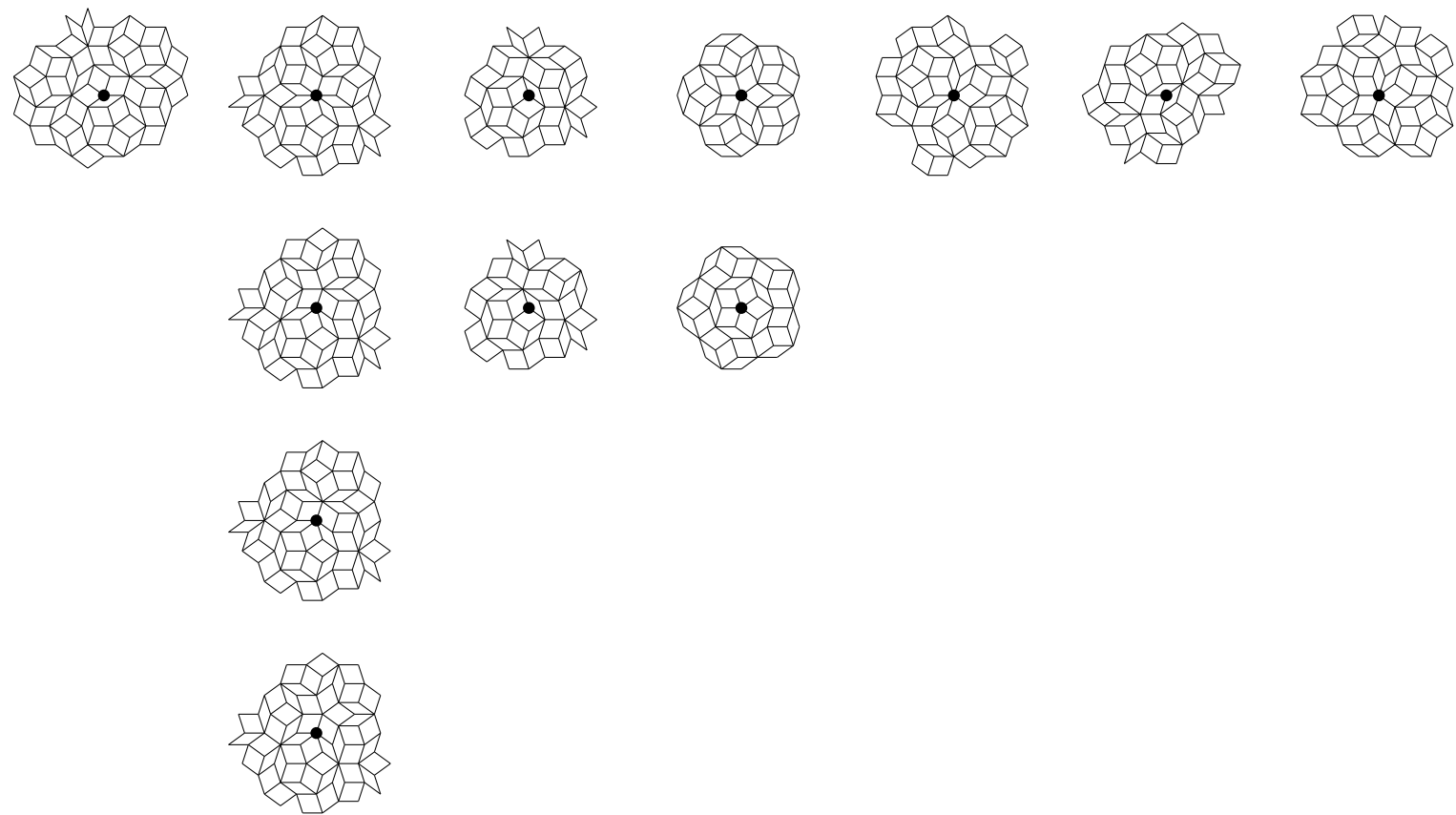

FIG. 5. Patches from the Penrose lattice used as input for the finite lattice method calculations. The columns contain all possible "boundary line fillings" of seven patches the central sites of which correspond to seven different vertex types.

\begin{tabular}{|c|c|c|c|c|c|c|c|c|c|}
\hline & $g_{3}$ & $g_{4}$ & $g_{5}$ & $g_{6}$ & $g_{7}$ & $g_{8}$ & $g_{9}$ & $g_{10}$ & $g_{11}$ \\
\hline$M(x)$ & -1.0832 & -0.1803 & -1.3736 & -3.656 & -10.0258 & -12.8017 & -11.2619 & -16.135 & -72.9404 \\
\hline$\chi(x)$ & 2.1026 & 0.3607 & 4.4234 & 16.0886 & 63.0069 & 110.5216 & 137.4134 & 241.4194 & 990.5163 \\
\hline$\chi^{(1)}(x)$ & -4.0136 & -0.7214 & -15.448 & -73.4949 & -404.9629 & -941.0482 & -1484.2237 & -3081.2191 & -12969.5517 \\
\hline$\chi^{(2)}(x)$ & -33.5551 & -5.861 & -94.3 & -405.998 & -2039.6444 & -4479.9119 & -6807.027 & -13838.423 & -58117.8824 \\
\hline & $g_{12}$ & $g_{13}$ & $g_{14}$ & $4 \quad g_{15}$ & $g_{16}$ & $g_{17}$ & $g_{18}$ & $g_{19}$ & $g_{20}$ \\
\hline$M(x)$ & -231.8001 & -493.5029 & -841.3831 & $1-1651.9956$ & -4125.5155 & -9628.4929 & -18432.4073 & -33133.1842 & -70657.8814 \\
\hline$\chi(x)$ & 3397.510 & 8427.160 & 17816.643 & 341630.163 & 3110568.346 & 277554.684 & 621098.121 & 1362853.02 & 3231583.007 \\
\hline$\chi^{(1)}(x)^{>}$ & * $\quad-49115$ & -139934 & -350428 & -944924 & -2738982 & -7510844 & -19047918 & -47924567 & -125178572 \\
\hline$\chi^{(2)}(x)$ & -217789 & -612298 & -1512009 & -4036132 & -11635964 & -31747510 & -79992423 & -200008381 & -520387269 \\
\hline
\end{tabular}

TABLE I. Expansion coefficients of the magnetisation $M(x)$, susceptibility $\chi(x)$ and its field derivatives $\chi^{(1)}(x)$, $\chi^{(2}(x)$ obtained from the finite lattice method.

\section{VERIFICATION OF CORRECTNESS OF THE COMPUTED EXPANSION}

There is a duality relation connecting the low temperature expansion of the Ising model on the lattice $\mathcal{G}$ to the high-temperature expansion on the dual lattice $\mathcal{D}$, which takes the following form:

$$
Z_{\mathcal{G}}(x, y)=\exp \beta(M J+N B) \tilde{Z}_{\mathcal{G}}(x, y)=2^{N}(\cosh \beta J)^{M}(\cosh \beta B)^{N} \tilde{Z}_{\mathcal{D}}(w, h)
$$

where the low temperature variables are $x=\exp \{-2 \beta J\}, y=\exp \{-\beta B\}$ and the high-temperature ones are $w=\tanh \{\beta J\}, h=\tanh \{\beta B\}$. In the field free case $h=0$ the high-temperature expansion of $\tilde{Z}_{\mathcal{D}}(w, 0)$ can be expressed by the square root of the determinant of a $2 M \times 2 M$ complex matrix [13,23], which for periodic lattices amounts to calculating a finite-dimensional determinant the dimension of which is of the order of the size of the unit cell. Therefore the free energy expansion in variable $x$ can be calculated by taking logarithms of equation (15).

$$
F=\lim _{N \longrightarrow \infty} \frac{1}{N} \log Z_{\mathcal{G}}(x)=\log 2-\frac{q}{4} \log \left(1-w^{2}\right)+\log \tilde{Z}_{\mathcal{D}}(w)
$$

where $q=\lim _{N \rightarrow \infty} 2 M / N$ is the mean coordination number. The expansion of the last term on the right-hand side 


$$
\log \tilde{Z}_{\mathcal{D}}(w)=\sum_{n=3}^{\infty} g_{n} w^{n}
$$

is obtained from Kac-Ward determinants for large enough PAs of the Penrose lattice, see [13] for detailed explanation. In TABLE II we show the expansion coefficients $g_{n}$ for successive PAs together with the coefficients of $F_{0}(x)$ (see 13) obtained by the finite lattice method (FLM). The data for the highest approximants are quite close to these for the FLM; the relative discrepancies for $n=3, \ldots, 20$ are equal $-4.9 \%,-0.2 \%,-0.4 \%,-5.1 \%,-4.7 \%,-2.8 \%, 6.8 \%, 14.5 \%, 1 . \%,-1.6 \%,-6.4 \%,-9.3 \%,-11.1 \%,-9.4 \%,-8.2 \%$, $-8.4 \%,-11.3 \%,-13.1 \%$ and in most cases do not exceed ten percent. In addition both data sets depend on $n$ in a similar way. Indeed, assuming known values for the critical point $x_{c}=0.434269$ and the critical exponent $\alpha=2$ we define the sequence $r_{n}$ in the following way:

$$
r_{n}=g_{n} / g_{n-1}-1 / x_{c}(1-(\alpha+1) / n)
$$

This sequence approaches zero $r_{n} \longrightarrow 0$ for large $n$, see pages 187-199 in [19]. If we now compare the sequences from both the PA coefficients and the FLM coefficients we see that the relative discrepancies except for $\mathrm{n}=5,10,12$ are all smaller than ten percent as well.

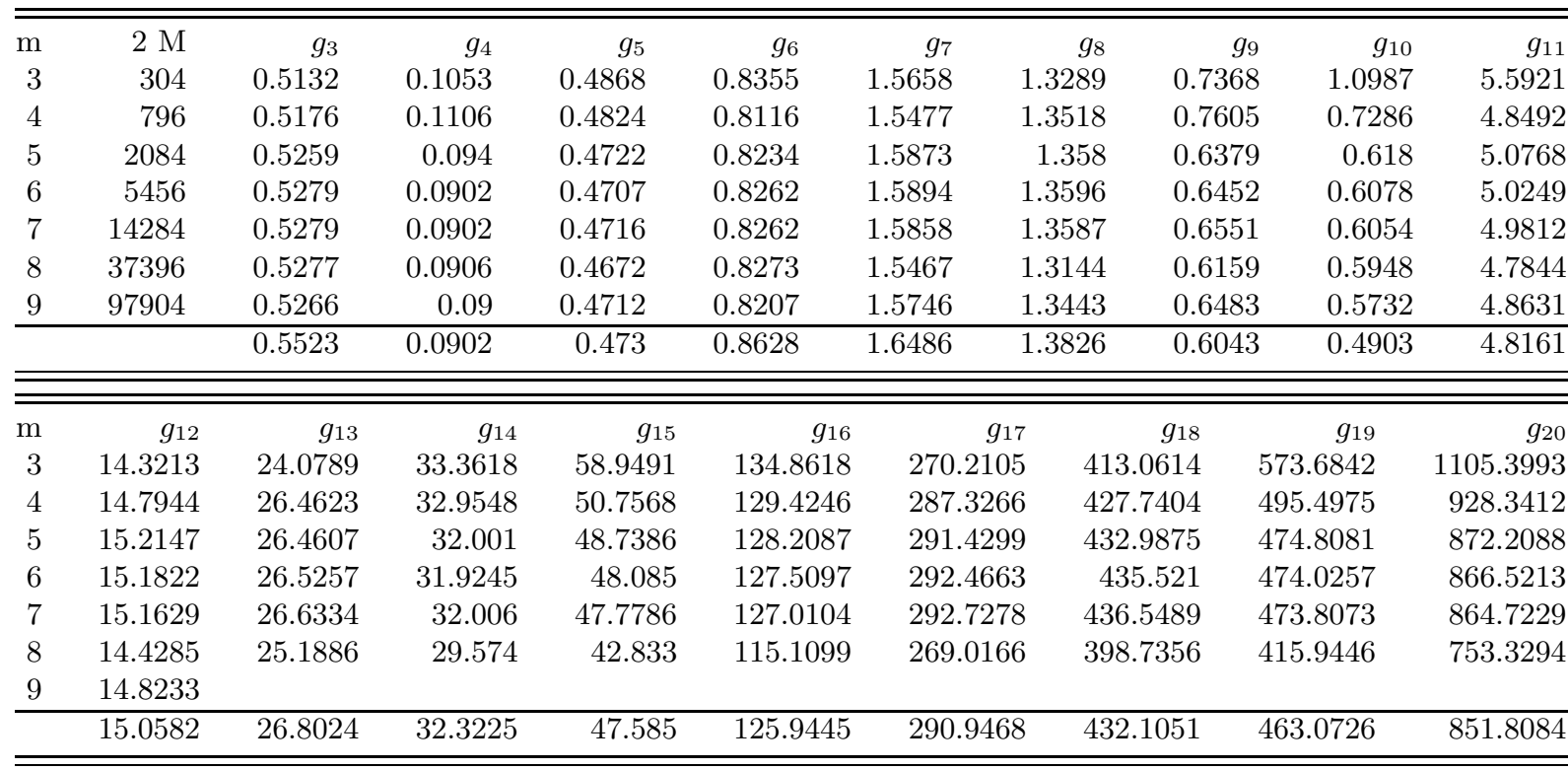

TABLE II. Expansion coefficients for free energy (17) for periodic approximants $m=3, \ldots, 9$ of the dual Penrose lattice with $M$ edges in the unit cell. Underneath the solid line coefficients obtained by the finite lattice expansion. The numbers $g_{n}$ approach the expansion coefficients for the dual Penrose lattice when $m \longrightarrow \infty$.

The lowest coefficients of our expansions can be also calculated exactly by counting graphs on the dual Penrose lattice. Here we confine ourselves to the free energy and the magnetisation expansions. We compute their first four nonzero coefficients and show that they are indeed close to those from tables TABLE 1 and TABLE [1]. We start from the low temperature expansion

$$
x^{M / 2} y^{N} Z_{\mathcal{G}}(x, y)=\tilde{Z}_{\mathcal{G}}(x, y)=\sum_{n, m} h_{n, m} x^{n} y^{2 m}
$$

with $h_{n, m}$ counting graphs, in general multicomponent graphs, from dual lattice consisting of $m$ sites and $n$ bonds on the perimeter. It is readily seen from figures (FIG.6, FIG.7, FIG.8 and FIG.9) that for the Penrose lattice the non zero coefficients take following values:

$$
\begin{array}{cc}
h_{3,1}=(7-4 \tau) N & h_{4,1}=(-8+5 \tau) N \\
h_{5,1}=(10-6 \tau) N & h_{5,2}=(-16+10 \tau) N \\
h_{6,1}=(-21+13 \tau) N & h_{6,3}=(-8+5 \tau) N \\
h_{7,1}=(13-8 \tau) N & h_{6,2}=(22-13 \tau) N+(7-4 \tau) N((7-4 \tau) N-1) \\
h_{7,3}=(79-48 \tau) N h_{7,2}=(-63+39 \tau) N+(7-4 \tau)(-8+5 \tau) N^{2}
\end{array}
$$


Let us notice that to coefficients $h_{6,2}$ and $h_{7,2}$ contribute also disjoint, two-component graphs thus the coefficients are second degree polynomials in $N$.

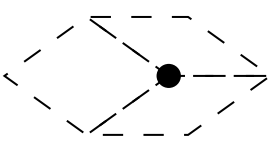

$(5-3 \tau) \mathrm{N}$

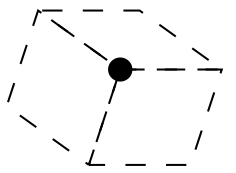

$(2-\tau) \mathrm{N}$

FIG. 6. Graphs from Penrose lattice contributing to the coefficient $h_{3,1}$ and their embedding numbers expressed through $\tau=(\sqrt{2}-1) / 2$. The dual graphs are constructed by connecting midpoints of rhombi abutting at bonds terminated by filled circles.
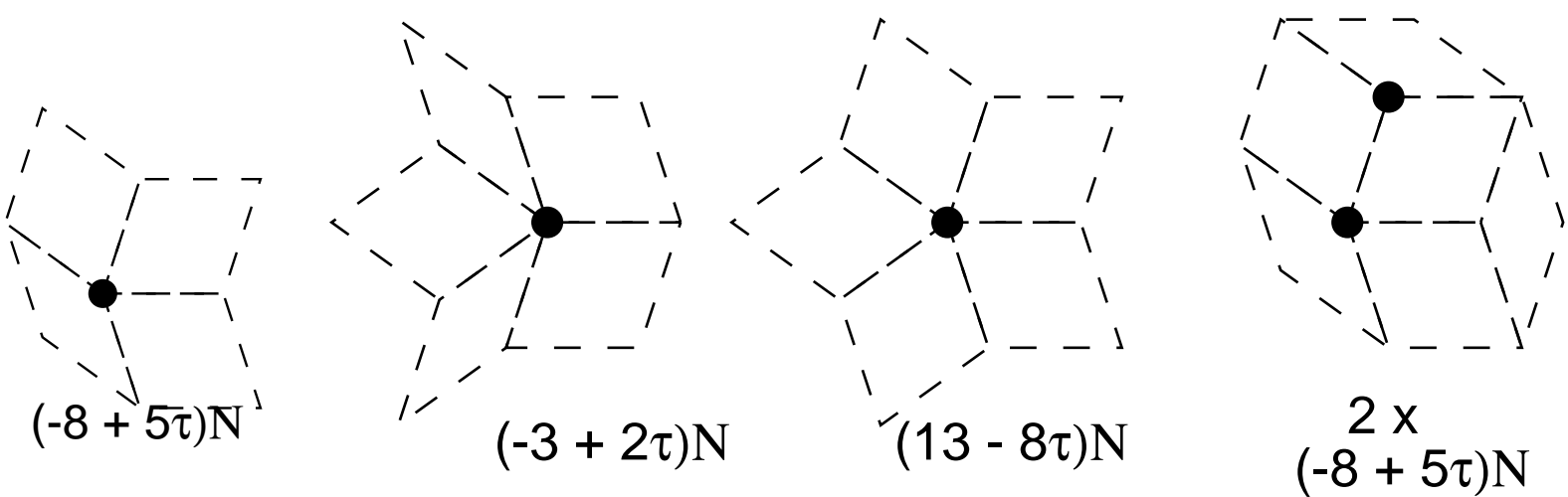

FIG. 7. The same as above corresponding to coefficients $h_{4,1}$ (three on the left) and $h_{5,1}$ (last on the right).
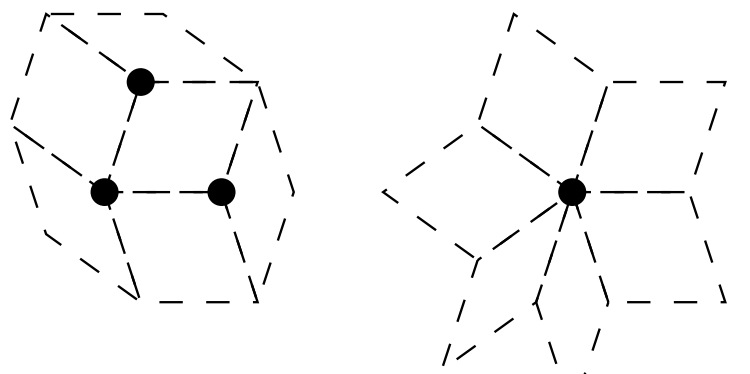

$$
(-8+5 \tau) \mathrm{N}
$$

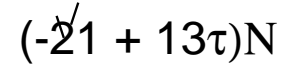

FIG. 8. Graphs contributing to the coefficients $h_{6,3}$ (left) and $h_{6,1}$ (right). 

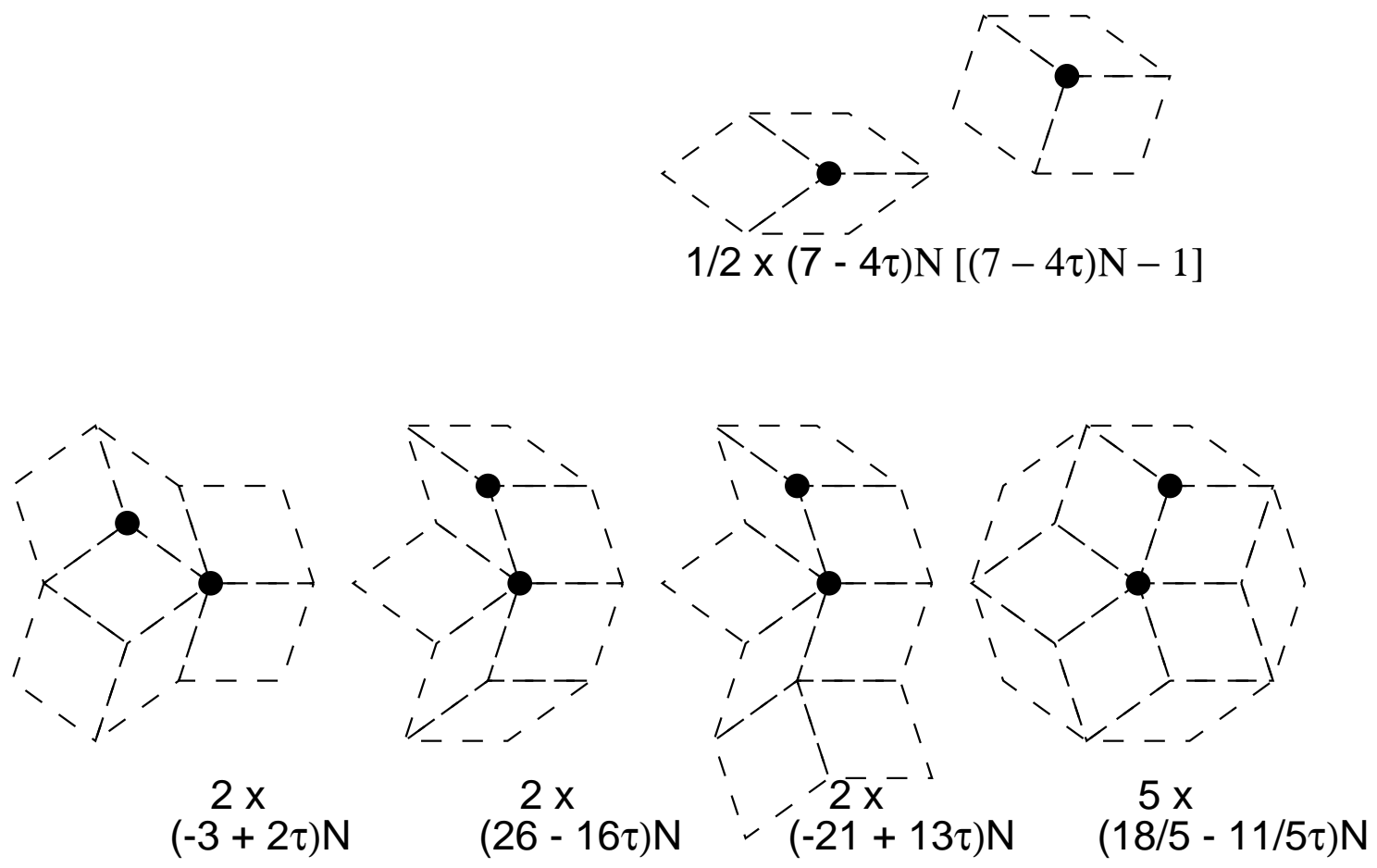

FIG. 9. Graphs contributing to the the coefficient $h_{6,2}$.

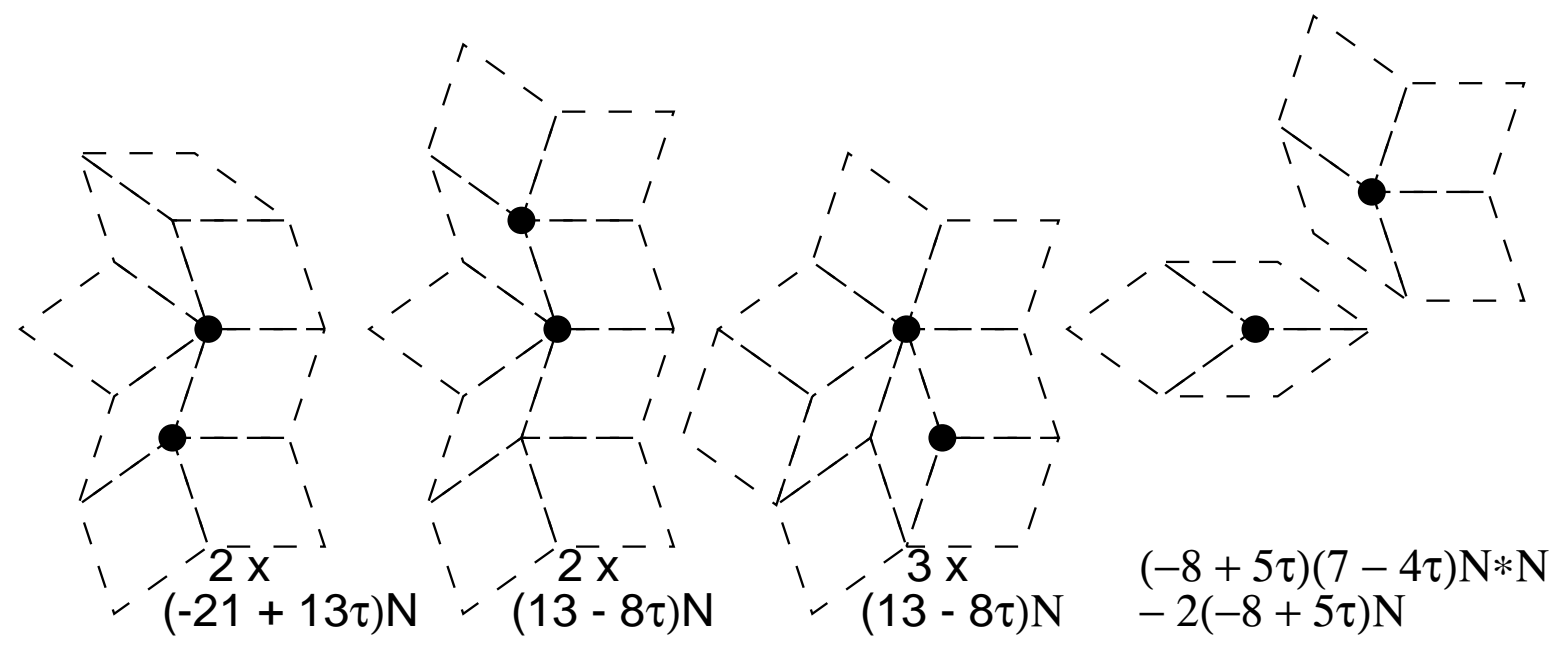

FIG. 10. Graphs contributing to the coefficient $h_{7,2}$. 

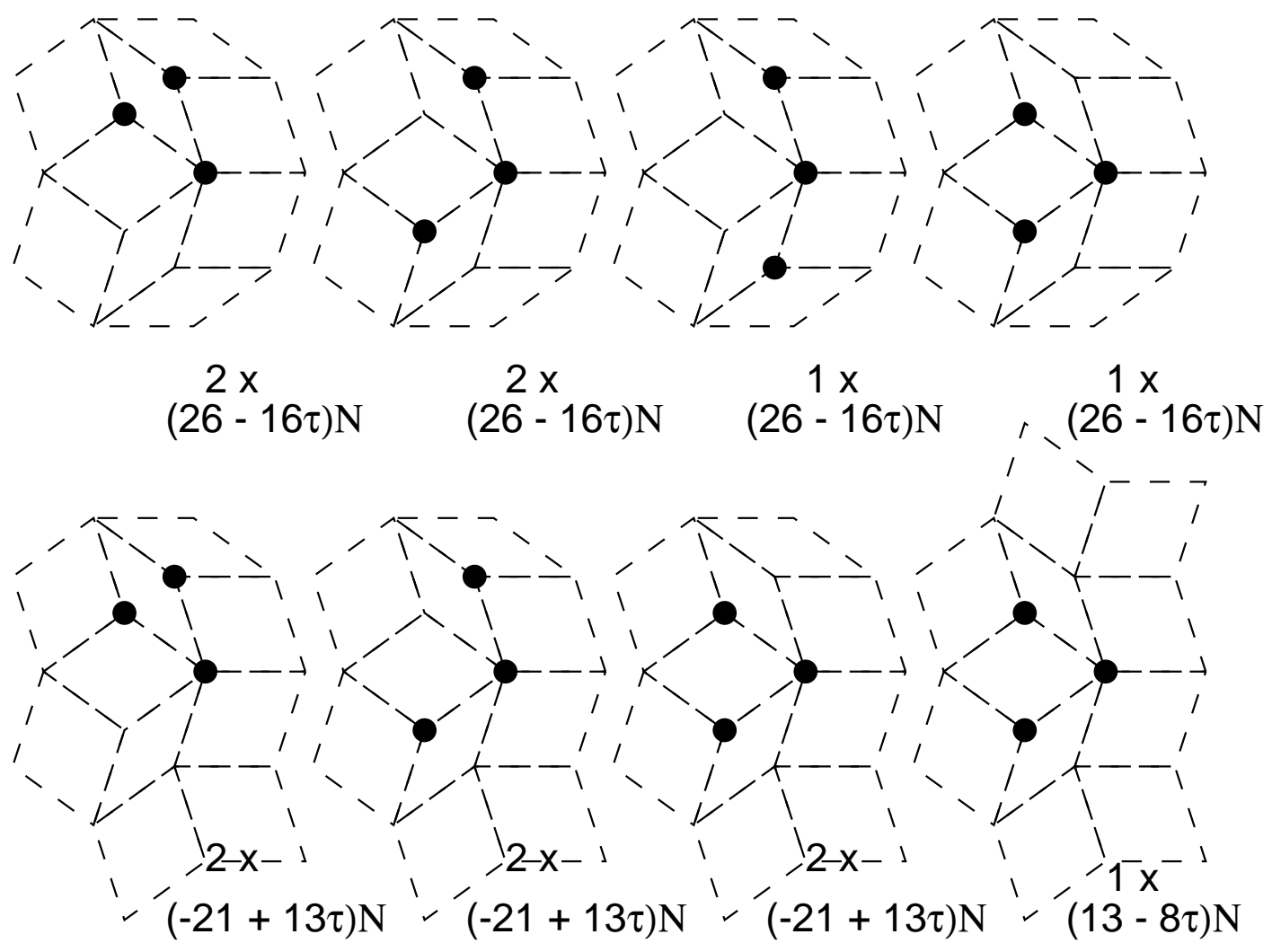

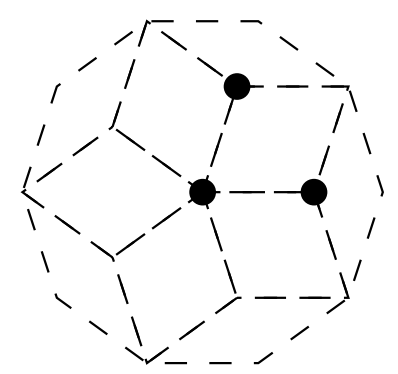

$5 \mathrm{x}$

$(18 / 5-11 / 5 \tau) \mathrm{N}$

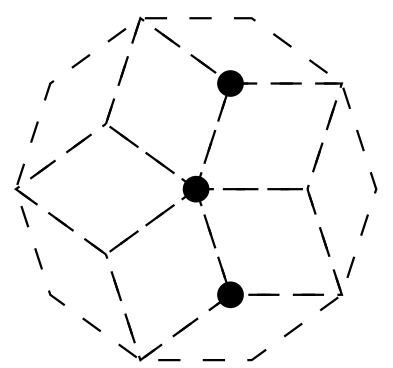

$5 \mathrm{x}$

$(18 / 5-11 / 5 \tau) \mathrm{N}$

FIG. 11. Graphs contributing to the coefficient $h_{7,3}$.

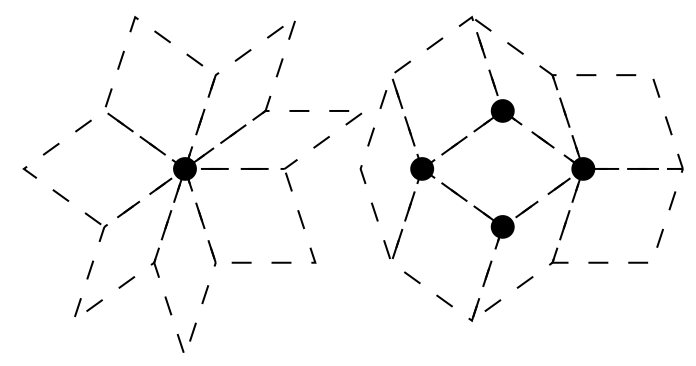

$$
(13-8 \tau) \mathrm{N} \quad(-8+5 \tau) \mathrm{N}
$$

FIG. 12. Graphs contributing to the coefficients $h_{7,1}$ (left) and $h_{7,4}$ (right). 
If we now insert the coefficients from (21) into the definitions of the magnetisation $M(x)$ and the susceptibility $\chi(x)$

$$
\begin{aligned}
& M(x)=\left.\frac{1}{N} \frac{d \log \left[Z_{\mathcal{G}}(x, y)\right]}{d B}\right|_{B=0}=1-\left.\frac{1}{N} y \frac{d \log \left[\tilde{Z}_{\mathcal{G}}(x, y)\right]}{d y}\right|_{y=1} \\
& \chi(x)=\left.\frac{1}{N} \frac{d M(x)}{d B}\right|_{B=0}=\left.\frac{1}{N} y \frac{d}{d y} y \frac{d \log \left[\tilde{Z}_{\mathcal{G}}(x, y)\right]}{d y}\right|_{y=1}
\end{aligned}
$$

we obtain following expansions:

$$
\begin{aligned}
F(x) & =0.5279 x^{3}+0.0902 x^{4}+0.4721 x^{5}+0.8262 x^{6}+1.58359 x^{7}+O\left(x^{8}\right) \\
M(x) & =1-1.0557 x^{3}-0.1803 x^{4}-1.3049 x^{5}-3.4164 x^{6}-9.25233 x^{7}+O\left(x^{8}\right) \\
\chi(x) & =2.1115 x^{3}+0.3607 x^{4}+4.0526 x^{5}+14.6099 x^{6}+55.6843 x^{7}+O\left(x^{8}\right) \\
\chi^{(1)}(x) & =4.2229 x^{3}+0.7214 x^{4}+13.8761 x^{5}+64.6563 x^{6}+341.449 x^{7}+O\left(x^{8}\right)
\end{aligned}
$$

which conform quite well to the values from TABLE I. Indeed, the relative differences between both sets of coefficients do not exceed ten percent in any case.

\section{ASYMPTOTIC ANALYSIS OF THE SERIES EXPANSIONS}

Now the problem consists in extracting critical exponents from the obtained expansions. The simplest approach, the ratio method, in which one examines the asymptotically linear dependence of ratios $g_{n} / g_{n-1}$ (18) on $1 / n$ and obtains $x_{c}$ and $\alpha$ from linear regression, is inapplicable in this case because of the slow convergence of series. Indeed, the residues $r_{n}(18)$ are much larger than those for the square lattice and alternate in sign, see figures FIG.13 and FIG.14, what makes the asymptotic analysis difficult. This approach requires knowledge of $x_{c}$ which is known from other works [6], [24] only with a limited accuracy. Applying the Padé method gives much more satisfactory results. Assuming that our thermodynamic functions $F(x)$ behave in the vicinity of the critical point $x_{c}$ like $F(x) \simeq\left(1-x / x_{c}\right)^{-\alpha} A(x)$ it is readily seen that functions $G_{0}(x)$ and $G_{1}(x)$ behave asymptotically as follows

$$
\begin{aligned}
& G_{0}(x)=\frac{d}{d x}\left(\log \frac{d F(x)}{d x}\right) / \frac{d}{d x}(\log F(x)) \simeq \frac{\alpha+1}{\alpha}+O\left(x-x_{c}\right) \\
& G_{1}(x)=\left(x-x_{c}\right) \frac{d}{d x}(\log F(x)) \simeq \alpha+O\left(x-x_{c}\right)
\end{aligned}
$$

Constructing Padé approximants to $G_{0}(x)$ and $G_{1}(x)$ and evaluating them at $x=x_{c}=0.434269$ usually yields a reasonable estimation of $\alpha$, even if $x_{c}$ is only known with a moderate accuracy. Results of this analysis, shown in tables TABLE [II and TABLE [V] are fairly close to the square lattice exponents. The sequence of Padé approximants is quite stable, except for few cases marked by asterix. Whenever the square lattice results converged Penrose data did as well.

On the other hand we can compute biased estimates of $x_{c}$, assuming known values of critical exponents. Indeed, the appropriate poles of Padé approximants to the function $[F(x)]^{1 / \alpha}$ should give rapidly convergent sequence of estimates of $x_{c}$. These sequences for the magnetisation expansion are shown in TABLE $\mathrm{V}$. In most cases the data do not deviate more than one percent from the exact values $x_{c}=0.434269$ (Penrose lattice [14]) and $x_{c}=\sqrt{(2)}-1$ (square lattice).

We can therefore claim that the data supports the claim that the quasiperiodic Ising model under consideration belongs to the square lattice (Onsager) universality class. 

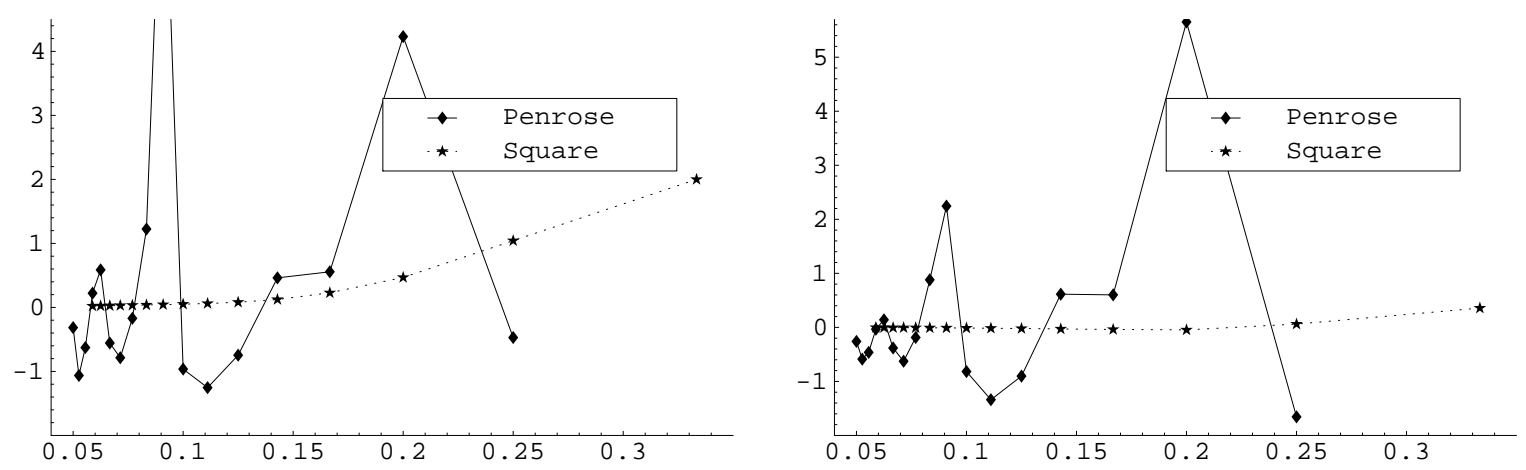

FIG. 13. Plots of residues $r_{n}(18)$ as a function of $1 / n$ for the free energy (left) and the magnetisation (right) on the Penrose and the square lattice respectively. In both cases we took the critical exponents $\alpha=2$ and $\beta=1 / 8$.
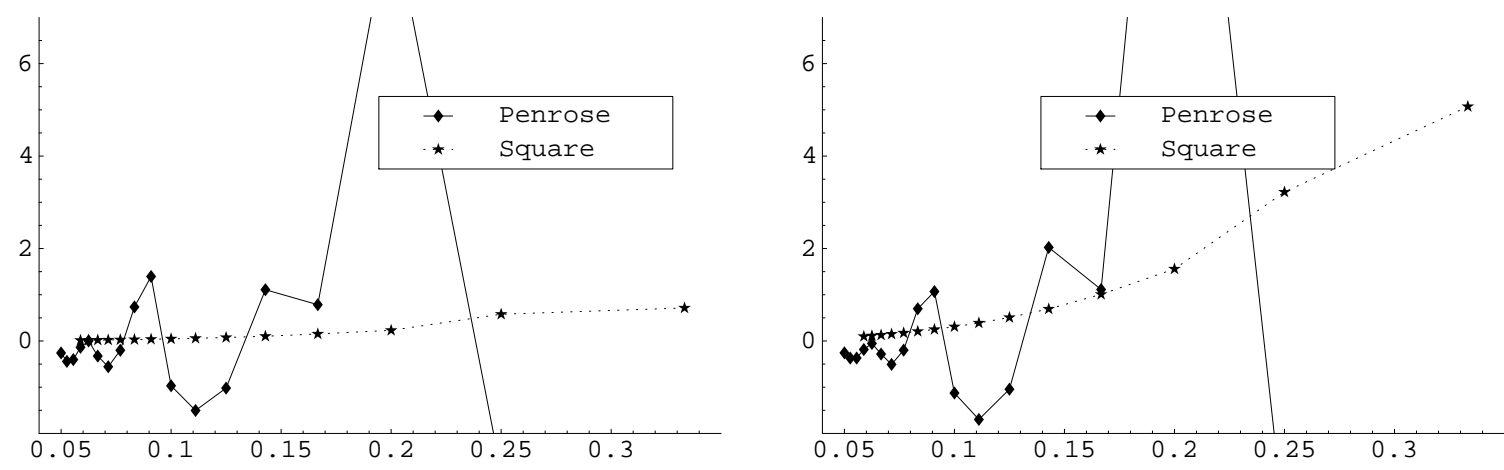

FIG. 14. As before for the susceptibility (left) and the field derivative of susceptibility (right). In both cases we took the critical exponents $\gamma=-7 / 4$ and $\delta=-29 / 8$.

\begin{tabular}{|c|c|c|c|c|c|c|c|c|c|c|c|c|}
\hline \multirow[t]{3}{*}{$\mathrm{n}$} & \multicolumn{4}{|c|}{$[\mathrm{n}, \mathrm{n}-1]$} & \multicolumn{4}{|c|}{$\begin{array}{c}\text { Approximant } \\
{[\mathrm{n}, \mathrm{n}]}\end{array}$} & \multicolumn{4}{|c|}{$[\mathrm{n}, \mathrm{n}+1]$} \\
\hline & \multicolumn{2}{|c|}{ Penrose } & \multicolumn{2}{|c|}{ Square } & \multicolumn{2}{|c|}{ Penrose } & \multicolumn{2}{|c|}{ Square } & \multicolumn{2}{|c|}{ Penrose } & \multicolumn{2}{|c|}{ Square } \\
\hline & $G_{0}(x)$ & $G_{1}(x)$ & $G_{0}(x)$ & $G_{1}(x)$ & $G_{0}(x)$ & $G_{1}(x)$ & $G_{0}(x)$ & $G_{1}(x)$ & $G_{0}(x)$ & $G_{1}(x)$ & $G_{0}(x)$ & $G_{1}(x)$ \\
\hline 8 & $0.159^{*}$ & 0.128 & 0.125 & 0.125 & 0.144 & 0.126 & 0.125 & 0.125 & 0.126 & 0.126 & 0.125 & 0.125 \\
\hline 9 & $0.242^{*}$ & 0.126 & 0.125 & 0.125 & 0.125 & 0.126 & 0.125 & 0.125 & 0.126 & 0.127 & 0.125 & 0.125 \\
\hline 10 & 0.121 & 0.127 & 0.125 & 0.125 & 0.123 & 0.126 & 0.125 & 0.125 & 0.126 & 0.126 & 0.125 & 0.125 \\
\hline 11 & 0.121 & 0.126 & 0.125 & 0.125 & 0.125 & 0.126 & 0.125 & 0.125 & 0.126 & 0.124 & 0.125 & 0.125 \\
\hline 12 & 0.140 & 0.128 & 0.125 & 0.125 & 0.135 & 0.114 & 0.125 & 0.125 & 0.113 & 0.120 & 0.125 & 0.125 \\
\hline 13 & 0.139 & 0.121 & 0.125 & 0.125 & 0.115 & 0.118 & 0.125 & 0.125 & 0.113 & 0.122 & 0.125 & 0.125 \\
\hline 14 & $0.067^{*}$ & 0.128 & 0.125 & 0.125 & $0.042^{*}$ & $0.097^{*}$ & 0.125 & 0.125 & 0.131 & 0.137 & 0.125 & 0.125 \\
\hline 15 & $0.069^{*}$ & $0.154^{*}$ & 0.125 & 0.125 & 0.128 & 0.146 & 0.125 & 0.125 & 0.130 & 0.100 & 0.125 & 0.125 \\
\hline
\end{tabular}

TABLE III. Estimates of the magnetisation critical exponent $\beta$ by means of Padé approximants $[n, m]$ to functions $G_{0}(x)$ and $G_{1}(x)(23)$ constructed from the expansion to order 20 for the square- and Penrose lattice respectively. Entries marked by asterix differ strongly from square lattice exponents. 


\begin{tabular}{|c|c|c|c|c|c|c|c|c|c|c|c|c|}
\hline \multirow[t]{3}{*}{$\mathrm{n}$} & \multicolumn{12}{|c|}{ Approximant } \\
\hline & Penı & cose & $\mathrm{Squ}$ & & Penr & cose & $\mathrm{Sq}$ & lare & Penı & rose & $\mathrm{Squ}$ & are \\
\hline & $G_{0}(x)$ & $G_{1}(x)$ & $G_{0}(x)$ & $G_{1}(x)$ & $G_{0}(x)$ & $G_{1}(x)$ & $G_{0}(x)$ & $G_{1}(x)$ & $G_{0}(x)$ & $G_{1}(x)$ & $G_{0}(x)$ & $G_{1}(x)$ \\
\hline 8 & $-1.287^{*}$ & -1.855 & $-1.383^{*}$ & -1.743 & -1.469 & -1.778 & -1.741 & -1.740 & $-1.095^{*}$ & -2.230 & -1.741 & -1.741 \\
\hline 9 & $-1.313^{*}$ & -1.832 & -1.741 & -1.741 & $-1.397^{*}$ & -1.946 & -1.741 & $0.000^{*}$ & $-0.808^{*}$ & -2.244 & -1.699 & -1.585 \\
\hline 10 & $-1.299^{*}$ & -1.834 & -1.702 & -1.585 & -1.723 & -1.790 & -1.716 & -1.454 & -1.259 & -1.559 & -1.716 & -1.587 \\
\hline 11 & -1.431 & -1.851 & -1.716 & -1.587 & $-1.133^{*}$ & $-1.249^{*}$ & -1.716 & $0.000^{*}$ & $-1.088^{*}$ & -1.758 & -1.732 & -1.747 \\
\hline 12 & $-1.095^{*}$ & $-3.743^{*}$ & -1.738 & -1.747 & $-1.131^{*}$ & $-0.244^{*}$ & -1.736 & -1.747 & $-1.259^{*}$ & $-1.129^{*}$ & -1.736 & -1.747 \\
\hline 13 & -1.472 & -1.569 & -1.736 & -1.747 & -2.005 & $-0.956^{*}$ & -1.736 & $0.000^{*}$ & $13.636^{*}$ & $0.316^{*}$ & -1.757 & -1.748 \\
\hline 14 & $-0.336^{*}$ & $-0.752^{*}$ & -1.737 & -1.747 & $-4.638^{*}$ & $-2.848^{*}$ & -1.742 & -1.748 & $-4.610^{*}$ & $-2.432^{*}$ & -1.742 & -1.748 \\
\hline 15 & $-4.610^{*}$ & $-2.458^{*}$ & -1.742 & -1.748 & $-4.637^{*}$ & -1.924 & -1.742 & $-0.001^{*}$ & $-7.190^{*}$ & $-2.411^{*}$ & -1.743 & -1.749 \\
\hline
\end{tabular}

TABLE IV. As before for the susceptibility critical exponent $\gamma$.

\begin{tabular}{|c|c|c|c|c|c|c|}
\hline \multirow[t]{2}{*}{$\mathrm{n}$} & \multicolumn{2}{|c|}{$[\mathrm{n}, \mathrm{n}-1]$} & \multicolumn{2}{|c|}{$\begin{array}{l}\text { Approximant } \\
{[n, n]}\end{array}$} & \multicolumn{2}{|c|}{$[\mathrm{n}, \mathrm{n}+1]$} \\
\hline & Penrose & Square & Penrose & Square & Penrose & Square \\
\hline 8 & 0.434 & 0.414 & 0.462 & 0.414 & 0.437 & 0.414 \\
\hline 9 & 0.433 & 0.414 & 0.434 & 0.414 & 0.434 & 0.414 \\
\hline 10 & 0.434 & 0.414 & 0.434 & 0.414 & 0.434 & 0.414 \\
\hline 11 & 0.434 & 0.414 & 0.434 & 0.414 & 0.435 & 0.414 \\
\hline 12 & 0.433 & 0.414 & 0.446 & 0.414 & 0.437 & 0.414 \\
\hline 13 & 0.434 & 0.414 & 0.435 & 0.414 & 0.436 & 0.414 \\
\hline 14 & 0.431 & 0.414 & 0.438 & 0.414 & $0.457+0.018 * I$ & 0.414 \\
\hline
\end{tabular}

TABLE V. Biased estimates of the critical point $x_{c}=\exp \left(-2 \beta_{c}\right)$ obtained from the magnetisation expansion to order 20 for the square- and Penrose lattice respectively.

\section{CONCLUDING REMARKS AND OUTLOOK}

The aim of this work was to analyse quasiperiodic Ising models by means of graphical series expansions. We calculated low temperature expansions of the free energy, magnetisation and the susceptibility to order 20 and extracted the respective critical exponents. We note that we did not obtain exact values for the exponents. They are marked by errors, which however do not exceed $10 \%$ in most cases. This feature is rather unusual for series expansions on regular lattices where the coefficients are exact to a given order which is determined by the size of patches used for calculations. This deserves further comment. There are the following two sources of errors in our method:

1. There are always graphs which cannot be embedded in any of the patches used in the calculations.

2. The FLM consists in covering and probing the lattice with a finite set of patches. Since the lattice is irregular, in particular, not periodic there can always be "holes", i.e. groups of sites, associated with graphs on the dual lattice, which are not covered by any of the patches. Such incomplete covering with only one single patch is shown in figure 15. Consequently, even if the patches are large there can always be few small graphs which cannot be embedded into them and which produce an error in even the lowest order coefficients. 

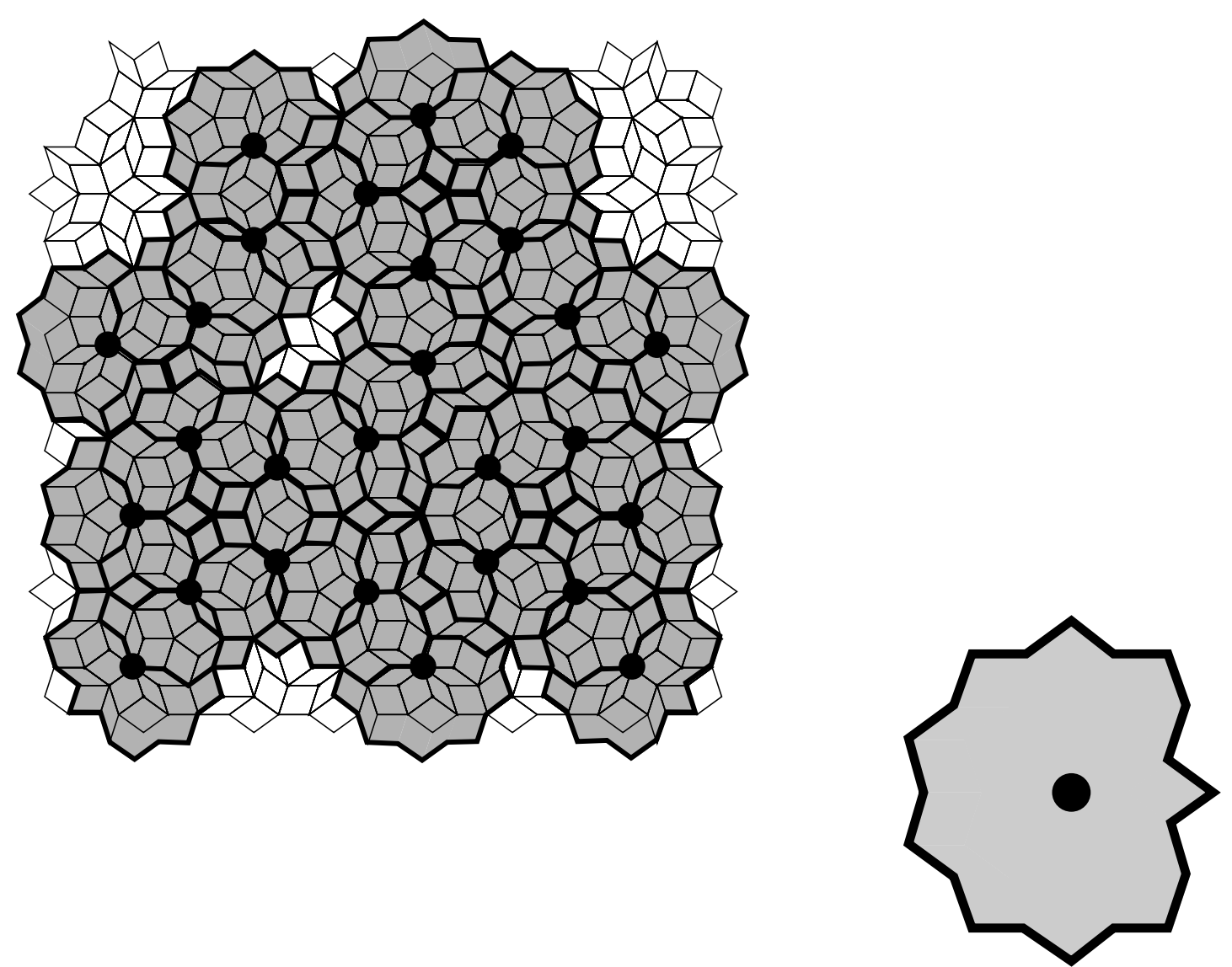

FIG. 15. Covering of a fragment of the Penrose lattice (left) with copies of a patch consisting of 18 thick and 10 thin rhombi (right). Central sites of patches are marked by black dots. One can see that the covering is not complete, there are holes not covered by any of the patches.

The next step in our research is to analyse the quasiperiodic $Q$-state Potts models, especially for $Q=$ 3,4 because the Harris-Luck criterion implies that quasiperiodic order should be strong enough to alter the critical behaviour in these cases. The main problem here consists in calculating partition functions for finite patches. This can be done, for instance, by improving the FLM [25] where the expansion for a particular $Q$ is obtained from partition functions with smaller $Q$ values. Another possibility is to use the Fortuin-Kasteleyn representation of the Potts model [17], expressing partition functions as a function of $Q$. Work in this direction is in progress.

\section{ACKNOWLEDGEMENTS}

P.R. thanks Des Johnston for discussions and an attentive proof reading of the paper. This work has been supported by a European Community IHP network HPRN-CT-1999-00161 "EUROGRID".

[1] Schechtman D, Blech I, Gratias D and Cahn J W 1984 Metallic phase with long-range orientational order and no translational symmetry Phys.Rev.Lett. 53 1951-1953

[2] Luck J M 1993 Europhys. Lett. 24 (5), 359-364

[3] Repetowicz P and Wolny J 1998 Diffraction pattern calculations for a certain class of N-fold quasilattices, $J$. Phys. A: Math. Gen. 31, 6873-6886

[4] Nischke K-P and Danzer L 1996 A construction of inflation rules based on $n$-fold symmetry, Discrete Comput. Geom. 15, No.2, 221-236 preprint Zentralblatt MATH 849.52016 
[5] Okabe Y and Niizeki K 1988 Monte Carlo simulation of the Ising model on the Penrose lattice, J.Phys.Soc.Jap. 57 16-19

[6] Sørensen E S, Jarić M V and Ronchetti M 1991 Ising model on the Penrose lattice: boundary conditions Phys.Rev. B 44 9271-9282

[7] Ledue D, Landau D P and Teillet J 1995 Static critical behaviour of the ferromagnetic Ising model on the quasiperiodic octagonal tiling Phys.Rev. B 51 12523-12530

[8] Redner O and Baake M 2000 Invaded cluster algorithm for critical properties of periodic and aperiodic planar Ising models J. Phys. A: Math. Gen. 33 3097-109

[9] Penrose R 1974 The role of aesthetics in pure and applied mathematical research Bull. Inst. Math. Appl. 10, 266-271

de Bruijn NG 1981 Algebraic theory of Penrose's non-periodic tilings of the plane I Indagationes Mathematicae (Proc. Kon. Ned. Akad. Wet. Ser. A) 84, 39-52

de Bruijn NG 1981 Algebraic theory of Penrose's non-periodic tilings of the plane II Indagationes Mathematicae (Proc. Kon. Ned. Akad. Wet. Ser. A) 84, 53-66

[10] Bose I 1987 Renormalization group (RG) study of the Ising model on higher-dimensional $(d>1)$ quasi-lattices Phys.Lett. A 123 224-226

[11] Aoyama H and Odagaki T 1987 Eight-parameter renormalization group for Penrose lattices J. Stat. Phys. 48 503-511

Aoyama H and Odagaki T 1988 Renormalization group analysis of the Ising model on two-dimensional quasi-lattices Int. J. Mod. Phys. B 2 13-35

[12] Abe R and Dotera T 1989 High temperature expansion for the Ising model on the Penrose lattice J.Phys.Soc.Japan 58 3219-3226

Dotera T and Abe R 1990 High temperature expansion for the Ising model on the dual Penrose lattice J.Phys.Soc.Japan 59 2064-2077

[13] Repetowicz P, Grimm U and Schreiber M 1999 High-temperature expansion of quasiperiodic Ising models, J. Phys. A: Math. Gen. 32, 4397-4418

[14] Repetowicz P, Grimm U and Schreiber M 2000 Planar quasiperiodic Ising models, Materials Science and Engineer. 294-296, 638-641

[15] Domb C 1989 Phase Transitions and Critical Phenomena vol 13 ed C. Domb, J. L. Lebowitz London: Academic Press

[16] Repetowicz P 2000 Theoretical investigations of magnetic and electronic properties of quasicrystals, PhD thesis, Technische Universität Chemnitz, Germany

[17] Wu F Y 1982 Rev. Mod. Phys. 54, 235-267

[18] Chen S, Ferrenberg A M and Landau D P 1995 Phys.Rev. E 521377

Janke W, Johnston D 2000 Ising and Potts Models on Quenched Random Gravity Graphs Nucl.Phys. B 578 681-698

Chatelain C, Berche P E and Berche B 1998 Second-Order Phase Transition Induced by Deterministic Fluctuations in Aperiodic Eight-State Potts Model 1999 Eur. Phys. J. B 7 439-449

[19] Domb C 1974 Phase Transitions and Critical Phenomena vol 3 ed C. Domb, J. L. Lebowitz London: Academic Press

[20] Hijmans J and De Boer J 1955 Physica XXI, 471-484

[21] Enting I G 1978 J.Phys. A: Math. Gen.,11 No. 3, 563-568

[22] Baake M, Grimm U, Repetowicz P and Joseph D Coordination sequences and critical points, in: Proc. of the 6th Int. Conf. on Quasicrystals (Tokyo 1997) Edit. S.Takeuchi und T.Fujiwara (Singapore: World Scientific), p.124-127, preprint cond-mat/9809110,

[23] Dolbilin N P, Zinovev Yu M, Shtanko M A and Shtogrin M K (1998) Russ. Math. Surv.,53 1346-1347

[24] Repetowicz P, Grimm U and Schreiber M (2000) Planar quasiperiodic Ising models Mat. Scien. and Enginner.,294-296 638-641

[25] Arisue H and Tabata K (1997) Low-temperature series for square lattice Potts model using the improved finite lattice method J.Phys. A.: Math.Gen.,30 3313-3327 